УДК 532.542.86. (088.8)

\title{
ТЕПЛОФИЗИЧЕСКАЯ МОДЕЛЬ АВТОКОЛЕБАНИЙ В РЕКУПЕРАТИВНЫХ ТЕПЛООБМЕННИКАХ ХОЛОДИЛЬНИКОВ ПРОМЫШЛЕННЫХ АГРЕГАТОВ
}

Басок Б.И., член-корреспондент НАН Украины, Гоцуленко В.В., доктор техн. наук

\section{Институт технической теплофизики НАН Украиньл, ул. Желябова, 2а, Киев, 03680, Украина}

Обгрунтований механізм Обоснован механизм отрицавід’ємного опору в потоці рідини тельного сопротивления в потоке при іiі стисненні i теплопідводі. жидкости при ее сжатии и теплоВикористовуючи емпіричне рів- подводе. Используя эмпирическое няння Тейта, одержана систе- уравнение Тэйта, получена система рівнянь нестаціонарного руху ма уравнений нестационарного рідини в пристроях із зосеред- движения жидкости в устройствах женими параметрами, визначені с сосредоточенными параметраïx періодичні автоколивальні ми, определены их периодические розв'язки.
The mechanism of negative resistance in a stream of a liquid is proved at her compression and a supply of heat. Using empirical Teta's equation, the system of the equations of non-stationary movement of a liquid in devices with the concentrated parameters is received, their periodic self-oscillatory decisions are determined.

Библ. 12. рис. 5.

Ключевые слова: тепловое сопротивление, автоколебания, предельный цикл, бифуркация, запаздывание парообразования.

\section{Введение}

Рекуперативные теплообменники используются в качестве холодильников при охлаждении фурм доменной печи, элементов ее конструкции, компрессоров и других энергетических устройств, охлаждаемых водой.

Известно [1], что при подводе теплоты к потоку жидкости или газа образуется тепловое сопротивление, которое наблюдается при движении идеальных жидкостей или газов [2]. Образование нисходящих ветвей зависимости $h_{\ell}(G)$ гидравлических потерь по длине [3] при ламинарном движении нагретого воздуха и теплового сопротивления $h_{\mathrm{T}}(G)[4]$, которое от режима движения не зависит, составляют механизмы возбуждения автоколебаний феномена Рийке. Кроме того, отрицательное тепловое сопротивление $h_{\mathrm{T}}(G)$ наряду с известным механизмом $\tau$ запаздывания сгорания, обоснованного Л. Крокко, является механизмом вибрационного горения, который оставался неизвестным. В явлении «поющего» пламени Хиггинса [5] наблюдается диаметральная противоположность изменения автоколебаний, самовозбуждающихся от запаздывания $\tau$ и механизма теплоподвода, когда волно- вое сопротивление $Z$ колебательного контура изменяется варьированием его акустической гибкости $C_{a}$.

Образование восходящей (неустойчивой) ветви на напорной характеристике $F(G)$ витка парообразования также происходит из-за наличия нисходящей ветви отрицательного сопротивления парообразования [6].

Механизмы, связанные с нисходящими ветвями сопротивлений различной природы, являются причиной возбуждения автоколебаний в явлении вольтовой дуги, при работе триода [7], в различных механических системах [8], но в гидропневмосистемах они оставались не обоснованными [9].

\section{Обоснование нисходящей ветви теплового со- противления в потоке жидкости}

Рассмотрим гидросистему (рис. 1), в которой под напором $H$ происходит истечение жидкости. Воспользовавшись уравнением Бернулли для сечений $1-1$ и $2-2$, определим скорость истечения идеальной жидкости при отсутствии ее нагрева в зависимости от напора $H$

$H=\frac{w_{\mathrm{x}}^{2}}{2 g}$

где $w_{\mathrm{X}}$ - скорость движения холодной жидкости. 


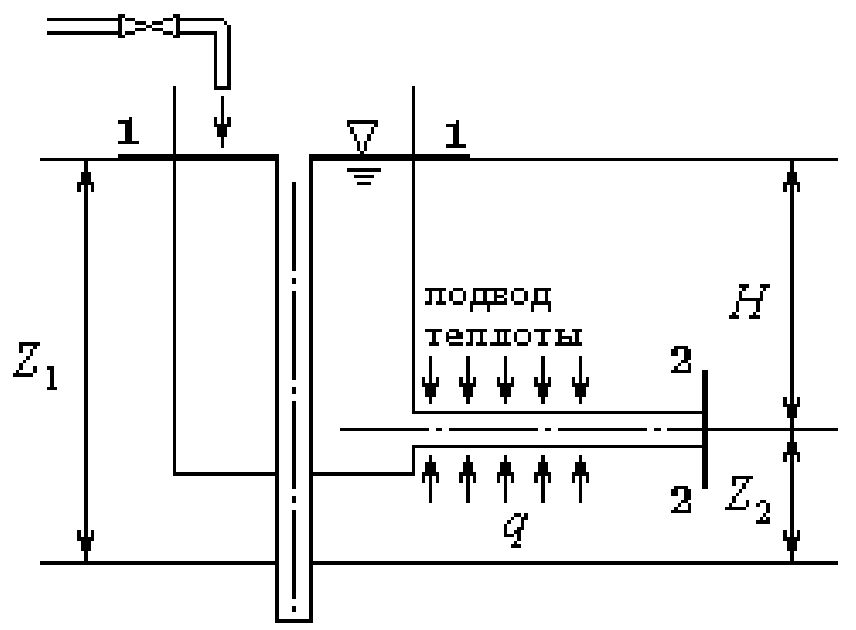

Рис. 1. К определению теплового сопротивления.

Подвод теплоты, при той же величине напора $H$, увеличивает скорость движения $w_{\mathrm{H}}$ нагретой невязкой жидкости, что приводит к возникновению сопротивления $h_{\mathrm{T}}(G)$. Уравнение Бернулли для сечений $1-1$ и $2-2$ при подводе теплоты следующее:

$Z_{1}+\frac{p_{0}}{\rho_{0} g}+\frac{w_{0}^{2}}{2 g}=Z_{2}+\frac{p_{0}}{\rho_{\mathrm{H}} g}+\frac{w_{\mathrm{H}}^{2}}{2 g}+h_{\mathrm{T}}(G)$.

Поскольку напор $H=Z_{1}-Z_{2}$, а скорость воды в емкости большого сечения $w_{0} \approx 0$ и избыточное давление $p_{0} \approx 0$, то из (2) следует

$h_{\mathrm{T}}(G)=H-\frac{w_{\mathrm{H}}^{2}}{2 g}$.

Исключив напор в уравнении (3), используя зависимость (1), приходим к соотношению

$h_{\mathrm{T}}(G)=\frac{w_{\mathrm{x}}^{2}-w_{\mathrm{H}}^{2}}{2 g}$, где $w_{\mathrm{X}}$ и $w_{\mathrm{H}}$ соответственно скорости холодной и нагретой жидкости.

Из уравнения неразрывности потока при постоянной нормальной площади сечения канала $\rho_{0} w_{\mathrm{X}}=\rho_{\mathrm{t}, 0} w_{\mathrm{H}}$ определим отношение скоростей $w_{\mathrm{H}} / w_{\mathrm{X}}$, и исключив его в уравнении (4), окончательно получим

$h_{\mathrm{T}}(G)=\frac{w_{\mathrm{x}}^{2}}{2 g}\left[1-\left(\frac{\rho_{0}}{\rho_{\mathrm{t}, 0}}\right)^{2}\right]$,

где $\rho_{0, \mathrm{t}}$ плотность нагретой жидкости при давлении $p_{0}$.

\section{Уравнения движения жсидкости в устройстве охлажсдения фурмы}

Скорость потока жидкости на входе в устройство охлаждения фурмы доменной печи (рис. 2) равна $w_{\mathrm{x}}=w_{0}$ Плотность технической воды на выходе из трубопровода 2 равна $\rho_{0, p}$, а после нагрева в фурме $\rho_{\mathrm{t}, \mathrm{p}}$.

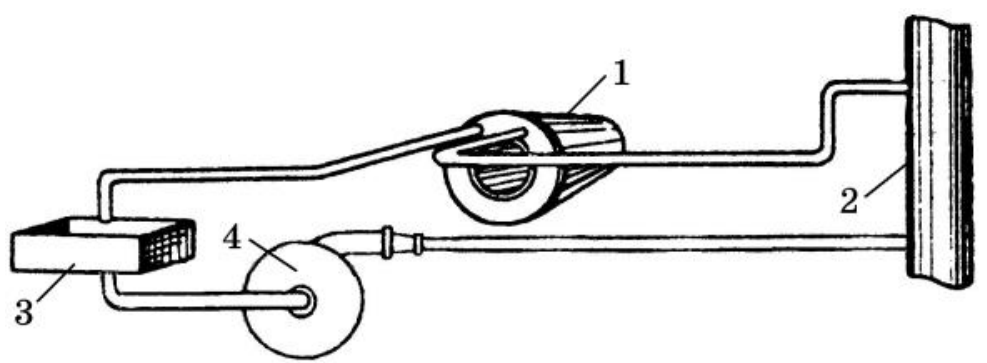

Рис. 2. Схема модели охлаждения фурмы доменной печи технической водой, где: 1 - фурма, 2 - трубопровод подачи технической воды, 3 - воронка, 4 - насос. 
При подводе теплоты к жидкости под давлением $p_{\mathrm{K}}$ на ее входе, тепловое сопротивление в фурме определяется выражением

$h_{\mathrm{T}}(G)=\frac{w_{0}^{2}}{2 g}\left[1-\left(\frac{\rho_{0, \mathrm{p}}}{\rho_{\mathrm{t}, \mathrm{p}}}\right)^{2}\right]$.

Воспользовавшись уравнением Тэйта [10] для жидкости $p=P_{*}\left(\frac{\rho}{\rho_{0}}\right)^{\mathrm{P}}$ определим ее плотность $\rho_{0, \mathrm{p}}$ на входе в фурму

$\rho_{0, \mathrm{p}}=\rho_{0}\left(\frac{p}{P_{*}}\right)^{1 / \Gamma}$,

где $P_{*}$ - давление при плотности $\rho_{0}$ жидкости на входе в насос является эмпирической постоянной, $p$ - давление на входе в фурму, г - показатель степени, также величина эмпирическая [10]. Из формулы (7) найдем величину $\rho_{0}$ и исключим ее в уравнении (6). Получим

$h_{\mathrm{T}}(G)=\frac{w_{0}^{2}}{2 g}\left[1-\left(\frac{\rho_{0}}{\rho_{\mathrm{t}, \mathrm{p}}}\right)^{2}\left(\frac{p}{P_{*}}\right)^{\frac{2}{\Gamma}}\right]$.

При нагреве жидкости с начальной плотностью $p_{0}$ на величину температуры $\Delta T$, ее плотность становится равной $\rho_{\mathrm{t}, 0}=\rho_{0}\left(1+\beta_{\mathrm{T}} \Delta T\right)^{-1}$, а при повышении давления на величину $\Delta p$, она определяется следующим выражением

$\rho_{\mathrm{t}, \mathrm{p}}=\rho_{0}\left(1+\beta_{\mathrm{T}} \Delta T\right)^{-1}\left(1-\beta_{\mathrm{p}} \Delta p\right)^{-1}$.

Поскольку $\beta_{\mathrm{p}} \Delta p=\frac{\Delta p}{K}$ является малой величиной, то плотность воды изменяется главным образом из-за температуры [11], т.е. $\rho_{\mathrm{t}, \mathrm{p}}=\rho_{0}\left(1+\beta_{\mathrm{T}} \Delta T\right)^{-1}$. Так как $\rho_{0}=\rho_{0, \mathrm{p}}$, то

$\rho_{\mathrm{t}, \mathrm{p}}=\rho_{0, \mathrm{p}}\left(1+\beta_{\mathrm{T}} \Delta T\right)^{-1}$.

Тогда тепловое сопротивление $h_{t}(G)$ после сжатия воды насосом в трубопроводе технической воды и ее нагреве в фурме, когда $\rho=\rho_{\mathrm{t}, \mathrm{p}}$, определяется в соответствии с уравнением (8) следующей формулой

$h_{\mathrm{T}}=\xi_{\mathrm{T}} \frac{\mathrm{w}_{0}^{2}}{2 \mathrm{~g}},\left[\left(\frac{P_{*}}{p}\right)^{\frac{1}{\Gamma}}\left(1+\beta_{\mathrm{T}} \Delta T\right)\right]^{2}, \Delta T-$ повышение
где $\xi_{\mathrm{T}}=1-\Delta \beta_{\mathrm{T}}-{ }^{2}$

температуры воды на выходе из фурмы, $\beta_{\mathrm{T}}-$ коэффициент теплового расширения, $w_{\mathrm{X}}=w_{0}$ скорость воды на входе в фурму. Для воды [10] $P_{*}=3250 \mathrm{\kappa г} / \mathrm{cm}^{2}, \Gamma=7.1$.
В задачах динамики гидросистем одной из определяющих величин является акустическая гибкость колебательного контура, характеризующая сжимаемость жидкости. Определим ее величину, воспользовавшись уравнением Тэйта. При этом следует учесть уравнение, определяющее модуль объемной упругости жидкости [10]

$$
K=\left.\rho_{0} \frac{\partial p}{\partial \rho}\right|_{\rho=\rho_{0}}=\Gamma P_{*} .
$$

Из уравнения Тэйта $p=P_{*}\left(\frac{\rho}{\rho_{0}}\right)^{\Gamma}[10]$ находим величину $\partial p / \partial \rho_{0, \mathrm{p}}$ и, воспользовавшись формулой $c=\sqrt{\frac{K}{\rho_{0}}}$, приходим к уравнению

$$
\frac{\partial p}{\partial \rho_{0, \mathrm{p}}}=P_{*}\left(\frac{\rho_{0, \mathrm{p}}}{\rho_{0}}\right)^{\Gamma-1} \frac{\Gamma \cdot c^{2}}{K},
$$

где $c$ - скорость звука.

Учитывая, что г $P_{*}=$ К из (11) следует, что

$\frac{\partial p}{\partial \rho_{0, \mathrm{p}}}=c^{2}\left(\frac{\rho_{0, \mathrm{p}}}{\rho_{0}}\right)^{\Gamma-1}$,

откуда, воспользовавшись уравнением Тэйта, приходим к следующему соотношению

$$
{\frac{\partial p}{\partial \rho_{0, \mathrm{p}}}}=c^{2}\left(\frac{p}{P_{*}}\right)^{1-\frac{1}{\Gamma}}
$$

из которого согласно (9) окончательно получаем

$$
\frac{\partial p}{\partial \rho_{0, \mathrm{p}}}=P_{*}\left(\frac{\rho_{0, \mathrm{p}}}{\rho_{0}}\right)^{\Gamma-1} \frac{\Gamma \cdot c^{2}}{K} .
$$

Запишем уравнение учитывающие накопление массы в емкости трубопровода с нагретой водой

$V \frac{d \rho_{\mathrm{t}, \mathrm{p}}}{d t}=G_{0}-G$

где $V$ - объем магистрали с нагретой водой, $G_{0}$ - расход подаваемый насосом, $G$ - выходящий из гидросистемы массовый расход, который определяется зависимостью $p=\varphi(G) \Leftrightarrow p=k_{\Gamma} G^{2}$ гидравлических потерь при движении нагретой воды.

Учитывая зависимость (12), уравнение (13) примет вид [6]

$$
\begin{aligned}
& C_{a} \frac{d p}{d t}=G_{0}-\varphi(p), \\
& \text { где } \quad C_{a}=\frac{V}{c^{2}\left(1+\beta_{\mathrm{T}} \Delta T\right)}\left(\frac{P_{*}}{p}\right)^{\frac{\Gamma-1}{\Gamma}}-
\end{aligned}
$$


емкость колебательного контура (рис. 2).

Уравнение движения холодной запишем в форме [6]

$L_{a} \frac{d G_{0}}{d t}=p_{\mathrm{\kappa}}-h_{\Sigma}\left(G_{0}\right)-p$,

где $\quad h_{\Sigma}\left(G_{0}\right)=h_{\mathrm{T}}\left(G_{0}\right)-h_{\ell}\left(G_{0}\right), \quad L_{a}=\ell_{0} / S, \ell_{0} \quad-$ длина трубопровода с холодной водой, $S$ - площадь поперечного сечения, $p_{\mathrm{K}}$ - давление в трубопроводе технической воды.

Полагая $F\left(G_{0}\right)=p_{\mathrm{K}}-h_{\mathrm{T}}\left(G_{0}\right)-h_{\ell}\left(G_{0}\right)$, уравнение движения (15) окончательно примет вид [12] $L_{a} \frac{d G_{0}}{d t}=F\left(G_{0}\right)-p$.

Система нелинейных уравнений (14), (16) определяет нестационарный режим движения нагреваемой жидкости в рекуперативных теплообменных аппаратах.
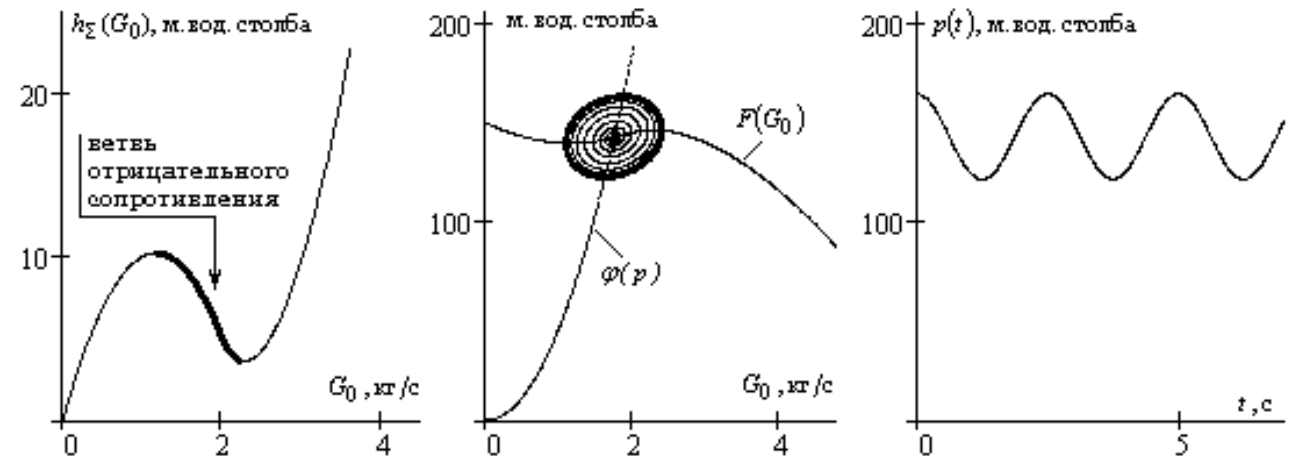

Рис. 3. Зависимость суммарного сопротивления $h_{\Sigma}\left(G_{0}\right)$, предельный цикл и форма автоколебаний р(t) в рассматриваемой модели (рис. 2) устройства охлажндения фурмы при $\xi=1,75$ кг/с.

При увеличении стационарного расхода $G_{0}=\xi \quad$ в гидросистеме (рис. 2) наблюдается переход от устойчивых стационарных режимов в области малых подач в неустойчивую область возбуждения автоколебаний значительных
Вязкостные потери $h_{\ell}\left(G_{0}\right)$ по длине трубопровода при движении воды на подогревательном участке для различных режимов движения определены в [6]. На рис. 3 приведена зависимость суммарных гидравлических потерь $h_{\Sigma}\left(G_{0}\right)$ с ветвью отрицательного сопротивления, которая образуется в зоне перехода ламинарного режима в турбулентный [6] и приводит к возбуждению автоколебаний [7-8]. Данные автоколебания теоретически получаются как периодические решения нелинейной автономной динамической системы (14), (16), форма которых и соответствующий им предельный цикл также приведены на рис. 3. Для определения предельного цикла и автоколебательных решений системы уравнений (14), (16) использовался алгоритм, разработанный в [3].
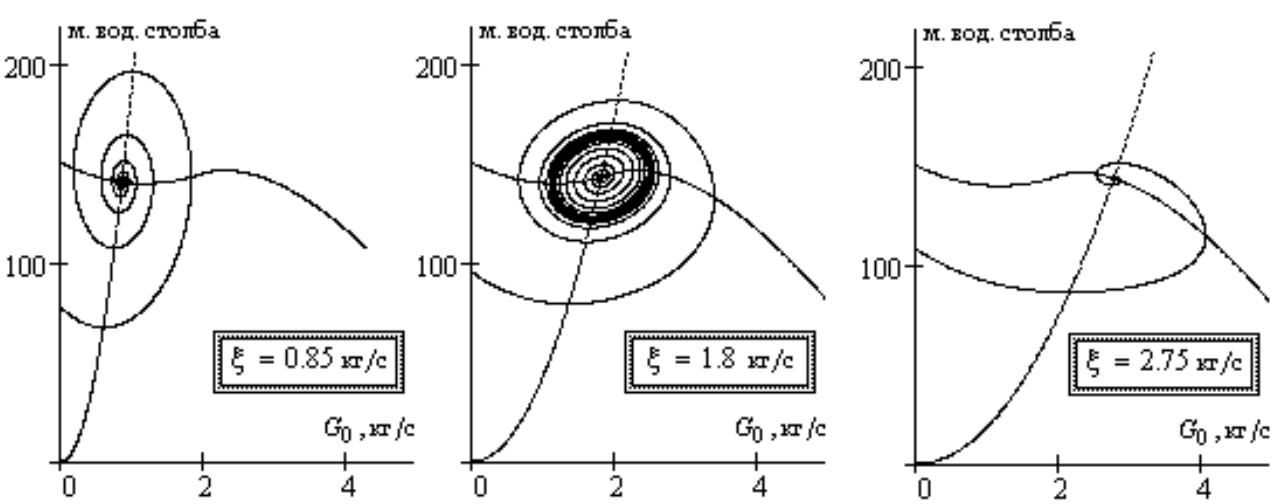

Рис. 4. Бифуркация предельного цикла при варьировании расхода Ц. 
Если на поверхности охлаждающей спиральной трубки в фурме наблюдается вскипание воды, то уменьшается скорость распространения звука в воде до минимального значения $c_{\min }$, а далее с увеличением степени сухости $x$ пара она возрастает до значения $c=\left.c\right|_{x=1}$. При этом возрастает акустическая гибкость $C_{a}=V / c^{2}$ и появляется транспортное запаздывание $\tau$ парообразования.
В этом случае уравнение сохранения массы (13) становится с запаздывающим аргументом и имеет вид

$$
C_{a} \frac{d p}{d t}=G_{0}(t-\tau)-\varphi(p) .
$$

Характер деформации предельного цикла с ростом запаздывания парообразования приведен на рис. 5.
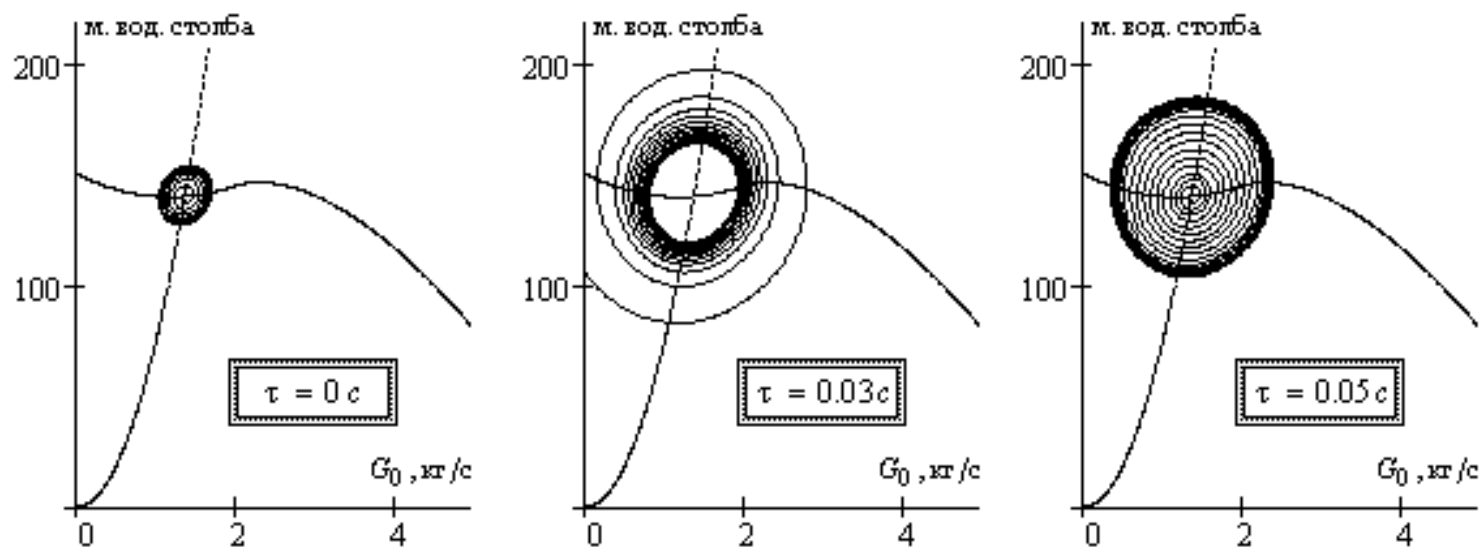

Рис. 5. Изменение предельного цикла с ростом $\tau$ запаздывания парообразования.

\section{Определение границы области устойчивости: условивия самовозбуждения автоколебаний}

Параметры стационарного режима течения жидкости определяются из системы дифференциально-разностных уравнений (16) - (17), полагая в ней $d G_{0} / d t=0$ и $d p / d t=0$.

Воспользовавшись разложением Тейлора

$G_{0}(t-\tau)=G_{0}(t)-\tau \frac{d G_{0}}{d t}+O\left(\tau^{2}\right)$

система (16) - (17), с точностью до величин порядка $O\left(\tau^{2}\right)$, запишется в следующей форме:

$L_{a} \frac{d G_{0}}{d t}=F\left(G_{0}\right)-p$,

$C_{a} \frac{d p t}{d t}=G_{0}-\frac{\tau}{L_{a}}\left(F\left(G_{0}\right)-p\right)-\varphi(p)$.

Далее более удобно перейти к безразмерным переменным:

$x=\frac{G_{0}-\xi}{\xi}, y=\frac{p-\eta}{\eta}$,

где введено обозначение $\eta=F(\xi)$. В переменных (19) система уравнений (18) запишется в следующем виде:

$\xi L_{\mathrm{a}} \frac{d x}{d t}=F(\xi+\xi x)-\eta(y+1)$, $\eta C_{\mathrm{a}} \frac{d y}{d t}=(x+1) \xi-\tau\left(\frac{F(\xi+\xi x)-\eta(y+1)}{L_{\mathrm{a}}}\right)-\xi(y+1)^{1 / 2}$.

Таким образом, характер устойчивости стационарного режима течения жидкости, в рассматриваемой задаче (рис. 2), окончательно сводится к исследованию устойчивости нулевого положения равновесия динамической системы (20). Согласно первому методу Ляпунова [7] исследования устойчивости рассмотрим матрицу Якоби системы (20), вычисленной в ее нулевом положении равновесия.

$\mathbf{J}=\left[\begin{array}{cc}\left.\frac{1}{L_{\mathrm{a}}} \frac{d F\left(G_{0}\right)}{d G_{0}}\right|_{G_{0}=\xi} & -\frac{\eta}{\xi L_{\mathrm{a}}} \\ \frac{\xi}{\eta C_{\mathrm{a}}}\left(1-\left.\frac{\tau}{L_{\mathrm{a}}} \frac{d F\left(G_{0}\right)}{d G_{0}}\right|_{G_{0}=\xi}\right. & \frac{1}{C_{\mathrm{a}}}\left(\frac{\tau}{L_{\mathrm{a}}}-\frac{1}{2} \frac{\xi}{\eta}\right)\end{array}\right]$.

Для определения критического времени запаздывания парообразования $\tau_{\text {кр }}$ необходимо предварительно вычислить корни характеристического уравнения: $\operatorname{det}(\mathbf{J}-\lambda \mathbf{E})=0$.

Раскрывая определитель (22), получаем

$\lambda_{1,2}=\frac{\operatorname{tr}(\mathbf{J}) \pm i \sqrt{4 \operatorname{det}(\mathbf{J})-\operatorname{tr}^{2}(\mathbf{J})}}{2}$ 
$\operatorname{tr}(\mathbf{J})=\left.\frac{1}{L_{\mathrm{a}}} \frac{d F\left(G_{0}\right)}{d G_{0}}\right|_{G_{0}=\xi}+\frac{1}{C_{\mathrm{a}}}\left(\frac{\tau}{L_{\mathrm{a}}}-\frac{1}{2} \frac{\xi}{\eta}\right)$,

$\operatorname{det}(\mathbf{J})=\left.\frac{1}{L_{\mathrm{a}} C_{\mathrm{a}}} \frac{d F\left(G_{0}\right)}{d G_{0}}\right|_{G_{0}=\xi}\left(\frac{\tau}{L_{\mathrm{a}}}-\frac{1}{2} \frac{\xi}{\eta}\right)+$

$+\frac{1}{L_{\mathrm{a}} C_{\mathrm{a}}}\left(1-\left.\tau \frac{d F\left(G_{0}\right)}{d G_{0}}\right|_{G_{0}=\xi}\right)$.

Таким образом, критическое время запаздывания парообразования определяется из условия: $\operatorname{Re}\left\{\lambda_{1,2}\right\}=0 \Leftrightarrow \operatorname{tr}(\boldsymbol{J})=\left.0 \Leftrightarrow \frac{1}{L_{\mathrm{a}}} \frac{d F(G)}{d G}\right|_{G=\xi}+$ $+\frac{1}{C_{\mathrm{a}}}\left(\frac{\tau_{\mathrm{\kappa p}}}{L_{\mathrm{a}}}-\frac{1}{2} \frac{\xi}{\eta}\right)=0$,

откуда окончательно получаем, что

$\tau_{\mathrm{\kappa p}}=\frac{1}{2} \frac{\xi L_{\mathrm{a}}}{F(\xi)}-\left.C_{\mathrm{a}} \frac{d F\left(G_{0}\right)}{d G_{0}}\right|_{G_{0}=\xi}$.

Следовательно, стационарный режим течения жидкости является устойчивым при выполнении неравенства $\tau<\tau_{\text {кр }}$. Соответственно при выполнении противоположного неравенства стационарный режим является неустойчивым и даже бесконечно малые его возмущения приводят к самовозбуждению автоколебаний.

\section{Выводы}

1. Используя эмпирическое уравнение Тэйта, обоснован механизм отрицательного сопротивления в жидкости при ее сжатии и теплоподводе.

2. Получена математическая модель автоколебаний, самовозбуждающихся из-за действия рассматриваемого механизма неустойчивости и установлен характер их изменения при варьировании стационарного массового расхода жидкости и запаздывания $\tau$ парообразования.

3. Установленная бифуркация предельного цикла при варьировании стационарного расхода жидкости позволяет осуществить управление амплитудой самовозбуждающихся автоколебаний.

\section{ЛИТЕРАТУРА}

1. Абрамович Г.Н. Прикладная газовая динамика / Абрамович Г. Н. - М.: Наука, 1969. - 824 с.

2. Раушенбах Б.В. Вибрационное горение /

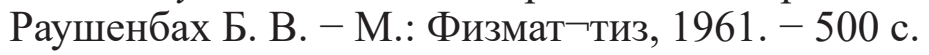

3. Гоиуленко В.B. Математическое моделирование особенностей феномена Рийке / В.В. Гоцуленко // Математическое моделирование, РАН. - 2004. - T. 16, № 9. - С. 23 - 28.

4. Гоиуленко В.В. Тепловое сопротивление как механизм возбуждения автоколебаний / В.В. Гоцуленко, В.Н. Гоцуленко // Сборник научн. трудов Днепродзержинского гос. техн. ун-та. - Днепродзержинск, 2009. - С. 95 - 100.

5. Гоиуленко В.В. Управление автоколебаниями колеблющегося пламени при одновременном действии механизмов их возбуждения / В. В. Гоцуленко, Б. И. Басок // Промышленная теплотехника. - 2009. - Т. 31, № 3. - С. 101 - 107.

6. Басок Б.И. Периодические движения теплоносителя в моделях элементов парогенераторов/ Б. И. Басок, В. В. Гоцуленко // Промышленная теплотехника. - 2010. - Т.32, № 4. - С. 33 -42 .

7. Меркин Д.Р. Введение в теорию устойчивости движения / Меркин Д. Р. - М.: Наука, 1971. $-312 \mathrm{c}$.

8. Стрелков С.П. Введение в теорию колебаний /Стрелков С. П. - М.: Наука, 1964. - 437 с.

9. Гершуни Г.3. Гидродинамическая неустойчивость. Изотермические течения / Г. З. Гершуни //Соросовский образовательный журнал. - 1997. - № 2. - C. $99-106$.

10. Зарембо Л.К. Введение в нелинейную акустику / Л.К. Зарембо, В.А. Красильников.М.: Наука, 1966. - 519 с.

11. Гинзбург И.П. Прикладная гидрогазодинамика. / Гинзбург И. П. - Л.: Изд-во Ленинградского. ун-та, 1958. - 338 с.

12. Басок Б.И. Расчет параметров автоколебаний в вертикальной камере горения воздухонагревателя доменной печи при неустойчивом горении/ Б. И. Басок, В. В. Гоцуленко// Теплоэнергетика. -2015 . - № 1. - С. $59-64$. 


\section{THERMOPHYSICAL MODEL OF SELF - OSCILLATIONS IN A RECUPERATIVE HEAT EXCHANGER REFRIGERATOR INDUSTRIAL AGGREGATES}

\section{Basok B.I., Gotsulenko V.V.}

Institute of Engineering Thermophysics of the National Academy of Sciences of Ukraine, vul. Zhelyabova, 2a, Kyiv-57, 03057, Ukraine

The mechanism of negative resistance in a stream of a liquid is proved at her compression and a supply of heat. Using empirical Teta's equation, the system of the equations of non-stationary movement of a liquid in devices with the concentrated parameters is received, their periodic self-oscillatory decisions are determined.

References 12, figures 5 .

Key words: thermal resistance, self-oscillation, limit cycle, bifurcation, the delay of evaporation.

1. Abramovich G.N. Prikladnaya gazovaya dinamika [Applied Gas Dynamics]. - Moscow: Nauka. - 1969. - 824 p. (Rus.)

2. Raushenbah B.V. Vibratsionnoe gorenie [Vibrating combustion]. - Moscow: Fizmattiz. 1961. - 500 p. (Rus.)

3. Gotsulenko V.V. Matematicheskoe modelirovanie osobennostej fenomena Rijke [Mathematical modeling of Rijke's phenomenon] // Matematicheskoe modelirovanie. - 2004. - V. 16, № 9. - P. 23 - 28. (Rus.)

4. Gotsulenko V.V., Gotsulenko, V.N. Teplovoe soprotivlenie kak mehanizm vozbuzhdeniya avtokolebaniy [Thermal resistance as a mechanism of excitation of oscillations]. // Sbornik nauchnyih trudov Dneprodzerzhinskogo gosudarstvennogo tehnicheskogo universiteta. - 2009. - V. 1 (11). - P. $95-100$. (Rus.)

5. Gotsulenko V.V., Basok B.I. Upravlenie avtokolebaniyami koleblyushhegosya plameni pri odnovremennom dejstvii mexanizmov ix vozbuzhdeniya [Control self-oscillations of the oscillating flame under the simultaneous action of their excitation mechanisms] // Promyshlennaya teplotekhnika. - 2009. - V. 31, № 3. - P. 101 107. (Rus.)

6. Basok B.I., Gotsulenko V.V. Periodicheskie dvizheniya teplonositelya $\mathrm{v}$ modelyax elementov parogeneratorov [Periodic movements of coolant in the steam generator model elements] // Promyshlennaya teplotekhnika. - 2010. - V.32, № 4. - P. $33-42$. (Rus.)

7. Merkin D.R. Vvedenie v teoriyu ustojchivosti dvizheniya [Introduction to the theory of stability of motion]. - Moscow: Nauka. - 1971. -312 p. (Rus.) 8. Strelkov S.P. Vvedenie v teoriyu kolebanij [Introduction to the theory of oscillations]. Moscow: Nauka. - 1964. - 437 p. (Rus.)

9. Gershuni G.Z. gidrodinamicheskaya neustojchivost. izotermicheskie techeniya [Hydrodynamic instability. Isothermal flow] // Sorosovskij obrazovatelnyj zhurnal. - 1997. - № 2. - P. 99 - 106. (Rus.)

10. Zarembo I.K. Vvedenie $\mathrm{v}$ nelinejnuyu akustiku [Introduction to nonlinear acoustics]. Moscow: Nauka. - 1966. - 519 p. (Rus.)

11. Ginzburg I. P. Prikladnaya gidrogazodinamika [Applied fluid dynamics]. - Leningrad.: izd-vo Leningradskogo un-ta. - 1958. - 338 p. (Rus.)

12. Basok B.I., Gotsulenko V.V. Raschet parametrov avtokolebanij $\mathrm{V}$ vertikalnoj kamere goreniya vozduxonagrevatelya domennoj pechi pri neustojchivom gorenii [Calculating the parameters of self-oscillations in the vertical combustion chamber of the blast-furnace air heater during unstable combustion] // Teploenergetika. 2015. - № 1. - P. 59 - 64. (Rus.)

Получено 21.03.2016 Received 21.03.2016 